\title{
Análise espectral segmentar no seguimento de pacientes revascularizados
}

\author{
Sequential spectrum analysis in the follow-up of revascularized patients
}

\author{
Domingos de Morais Filho, Fernando Thomazinho, Fernando Motta, Igor Schincariol Perozin, \\ Wander Eduardo Sardinha, José Manuel da Silva Silvestre, Oswaldo Palma, \\ Rodrigo Gomes de Oliveira*
}

\begin{abstract}
Resumo
Contexto: Pacientes tratados por revascularização arterial (enxertos e angioplastias) nos membros inferiores acometidos por doença arterial oclusiva periférica foram seguidos por até 2 anos pós-tratamento usando ultrassom vascular (análise espectral segmentar, AES).
\end{abstract}

Objetivo: Demonstrar que a AES pode ser utilizada no seguimento dos pacientes tratados por doença arterial oclusiva periférica.

Métodos: As medidas de AES realizadas foram: velocidade sistólica de pico, índice de pulsatilidade e forma da onda de velocidade de fluxo. Essas foram comparadas em cada paciente no pré e pós-tratamento (com intervalos de 3 meses) para diagnóstico de perviedade.

Resultados: Medidas realizadas no pós-operatório nas artérias imediatamente distais aos segmentos tratados mostraram aumento consistente de velocidade sistólica de pico e índice de pulsatilidade com mudanças na forma da onda de velocidade de fluxo de unifásica para bi ou trifásica. A velocidade sistólica de pico e o índice de pulsatilidade aumentaram respectivamente em 92,26 e 98,2\% (tratamentos no segmento aorto-ilíaco), em 112,83 e $62,39 \%$ (tratamentos no segmento fêmoro-poplíteo) e em 149,08 e $28,8 \%$ (tratamentos no segmento poplíteo-tibial). Tais mudanças nos padrões de velocidade de fluxo ocorreram em todos os pacientes e permaneceram quase inalteradas enquanto os tratamentos estivessem pérvios. Quando ocorria falência nos tratamentos (oclusões ou estenoses hemodinamicamente significantes), os parâmetros caíam a níveis similares aos de antes do tratamento. Se a falência do tratamento era corrigida por nova revascularização (enxerto ou angioplastia), os parâmetros de AES voltavam a se comportar como após o tratamento inicial.

Conclusão: A AES pode ser usada no seguimento dos pacientes com revascularização dos membros devido a doença arterial oclusiva periférica, demonstrando tanto a perviedade quanto a falência do tratamento.

Palavras-chave: Aterosclerose, eco-Doppler, ultrassom.

\section{Introdução}

Como a doença arterial oclusiva periférica (DAOP) tem caráter segmentar e pode apresentar lesões concomitantes, deve-se conhecer a fundo sua distribuição anatômi-

\begin{abstract}
Background: Patients treated for peripheral arterial occlusive disease with lower limb revascularization (angioplasty or grafts) were followed up for a 2-year period after treatment with vascular ultrasound (segmental spectrum analysis, SSA).
\end{abstract}

Objective: To demonstrate that SSA can be used in the follow-up of patients treated for peripheral arterial occlusive disease.

Methods: The following SSA measurements were performed: peak systolic velocity (PSV), pulsatility index (PI), and flow velocity waveform (FVW). These measurements were performed and compared for each patient during the pre- and post-treatment periods (with 3-month intervals) for diagnosis of vascular patency.

Results: Measurements performed postoperatively in the arteries immediately distal to the treated segments showed a significant increase in PSV and PI, with a change of the FVW from a monophasic to a biphasic or triphasic configuration. PSV and PI increased, respectively, 92.26 and $98.2 \%$ (intervention in the aortoiliac segment), 112.83 and $62.39 \%$ (intervention in the femoral-popliteal segment), and 149.08 and $28.8 \%$ (in the popliteal-tibial segment). Such changes in flow velocity patterns occurred in all patients and remained almost unaltered during the period of patient follow up. When treatment failed (hemodynamically significant occlusion or stenosis), parameters fell to levels similar to those observed prior to treatment. If treatment failure was corrected by new revascularization (angioplasty or grafts), SSA parameters returned to patterns observed after initial treatment.

Conclusion: SSA can be used in the follow-up of patients with lower limb revascularization due to peripheral arterial occlusive disease, demonstrating treatment patency and failure.

Keywords: Atherosclerosis, Doppler ultrasound, ultrasound.

ca bem como a importância hemodinâmica relativa das lesões presentes em cada caso. As mudanças nos padrões hemodinâmicos após o tratamento também precisam ser conhecidas para que se avalie a eficácia do tratamento e

\footnotetext{
* Hospital Universitário Estadual do Norte do Paraná (HURNP), Universidade Estadual de Londrina (UEL), Departamento de Clínica Cirúrgica, Setor de Cirurgia Vascular, Londrina, PR.

Não foram declarados conflitos de interesse associados à publicação deste artigo. Artigo submetido em 27.04.08, aceito em 30.03.09.

J Vasc Bras. 2009;8(2):112-124.

Copyright (C) 2009 by Sociedade Brasileira de Angiologia e de Cirurgia Vascular
} 
sua durabilidade. $\mathrm{O}$ ultrassom vascular tem-se mostrado como o melhor método para confirmação do diagnóstico inicial, bem como para a quantificação relativa dos segmentos envolvidos e para o seguimento DAOP, devido principalmente à sua capacidade de fornecer medidas objetivas passíveis de comparação temporal ${ }^{1-12}$. Um dos métodos para avaliação da DAOP pelo ultrassom vascular foi chamado de análise espectral segmentar (AES) ${ }^{13}$, que avalia os parâmetros da hemodinâmica arterial de maneira segmentar. O presente trabalho tem por objetivo determinar padrões de alterações hemodinâmicas baseadas nas medidas de AES nos diferentes arranjos de lesões da DAOP antes do tratamento e avaliar as mudanças de padrões após o tratamento nos casos em que esse foi bem sucedido e seu comportamento se houvesse falência do tratamento.

Os objetivos do trabalho foram:

- Demonstrar as mudanças nos parâmetros de AES usadas como exame de rastreamento na localização e quantificação de lesões hemodinamicamente significantes da DAOP nos membros inferiores;

- Correlacionar tais medidas com alterações da hemodinâmica local e os diferentes arranjos das lesões ateroscleróticas na DAOP usando AES;

- Comparar as medidas de AES feitas antes do tratamento com as feitas após os tratamentos e relacioná-las com as alterações ocorridas na hemodinâmica arterial após os tratamentos;

- Seguir os pacientes tratados com revascularização dos membros usando AES para definir padrões dos parâmetros e suas mudanças no decorrer do seguimento;

- Mostrar quais alterações dos parâmetros de AES ocorre no pós-tratamento da DAOP com revascularização arterial (enxertos ou angioplastias) e quais ocorrem se houver falência (oclusão ou estenoses hemodinamicamente significantes) destes tratamentos;

- Definir a mudanças dos parâmetros após a nova revascularização e comparar estes padrões com os medidos após o tratamento inicial.

\section{Métodos}

Entre janeiro de 2005 e dezembro de 2007, 205 membros foram tratados por isquemia arterial (em 193 pacientes) causada por DAOP nos membros inferiores (117 homens e
76 mulheres). Tais pacientes foram submetidos a revascularizações (angioplastias ou enxertos). O tratamento foi unilateral nos 193 casos e bilateral em 12 casos. Todos os pacientes foram tratados no setor de cirurgia vascular. O estudo foi aprovado pelo Comitê de Ética em Pesquisa envolvendo seres humanos da Universidade Estadual de Londrina (UEL) (parecer 172/05), em Londrina (PR). Todos os pacientes apresentavam isquemia crítica (categorias 5 e 6 ) da Society of Vascular Surgery/International Society of Cardiovascular Surgery (SVS/ISCVS) ${ }^{7}$. Todos os pacientes foram examinados por ultrassom vascular antes da angiografia e após revascularização (por enxerto ou angioplastia com ou sem stents) antes de receber alta. Após o tratamento, os pacientes foram acompanhados através de ultrassom vascular com AES em intervalos trimestrais. Todos os pacientes foram submetidos a angiografia previamente ao tratamento, para planejamento do tratamento e quando havia indicação de falência no mesmo.

Os pacientes foram categorizados em diferentes grupos de acordo com achados angiográficos, tais como portadores de lesões hemodinamicamente significantes (que necessitavam de revascularização por enxertos ou tratamentos endovasculares) em um ou mais segmentos arteriais. Os grupos formados foram: pacientes portadores de lesões unicamente no território aorto-ilíaco (grupo 1, Tabelas 1 e 2), portadores de lesões significativas apenas no trajeto fêmoro-poplíteo (grupo 2, Tabelas 3 e 4), portadores de lesões significantes somente no segmento poplíteo-tibial (grupo 3, Tabelas 5 e 6 ) e portadores de lesões significantes concomitantemente no território aorto-ilíaco e fêmoro-poplíteo (grupo 4, Tabelas 7 e 8 ).

O protocolo de exame de ultrassom vascular usado foi: com o paciente em decúbito dorsal, as artérias ilíacas externas, femorais comuns, tibiais anteriores e posteriores e fibulares eram examinadas, e, com o paciente em decúbito ventral, as artérias poplíteas foram examinadas. As medidas de AES descritas em estudo prévio ${ }^{13}$ foram realizadas em cada paciente na artéria femoral comum ou ilíaca externa (ao nível da prega inguinal), na artéria poplítea (no oco poplíteo), nas artérias da perna (tibial anterior, posterior), ao nível do tornozelo. Foram realizadas as seguintes medidas de análise espectral (Figura 1): tipo de onda de velocidade de fluxo, velocidade sistólica de pico (VSP) e índice de pulsatilidade (IP). A forma da onda de velocidade de fluxo foi classificada segundo suas fases (Figuras $1 \mathrm{e}$ 
Tabela 1 - Grupo 1: análise espectral na artéria femoral comum e ITB dos pacientes portadores de DAOP exclusivamente no território aorto-ilíaco pré e pós-tratamentos (médias)

\begin{tabular}{lcccccc}
\hline Aorto-ilíaco & Pré $(\mathrm{n}=65)$ & Pós $(1$ mês $)(\mathrm{n}=65)$ & 6 meses $(\mathrm{n}=53)$ & 12 meses $(\mathrm{n}=49)$ & 24 meses $(\mathrm{n}=46)$ & Pré/pós $(1$ mês $)(\%)$ \\
\hline ITB & 0,48 & 0,80 & 0,77 & 0,74 & 0,75 & 66,6 \\
VSP & 61,27 & 117,80 & 112,54 & 93,74 & 119,2 & 92,26 \\
IP & 2,23 & 4,42 & 4,30 & 3,33 & 4,37 & 98,2 \\
FOV & UBA & $\mathrm{B} / \mathrm{T}$ & $\mathrm{B} / \mathrm{T}$ & $\mathrm{B} / \mathrm{T}$ & $\mathrm{B} / \mathrm{T}$ & $\mathrm{B} / \mathrm{T}$ \\
\hline
\end{tabular}

$\mathrm{B}=$ bifásica; DAOP = doença arterial oclusiva periférica; FOV = forma da onda de velocidade de fluxo; IP = índice de pulsatilidade; ITB = índice tornozelo-braço; T = trifásica; UBA = unifásica de baixa aceleração; VSP = velocidade sistólica de pico.

Tabela 2 - Grupo 1: pacientes de DAOP exclusivamente no território aorto-ilíaco e tratados por angioplastia que apresentaram oclusões ou estenoses hemodinamicamente significantes no segmento aorto-ilíaco após os tratamentos (falência do tratamento) (médias)

\begin{tabular}{|c|c|c|c|c|c|c|c|c|c|}
\hline \multirow[b]{2}{*}{ Pacientes } & \multicolumn{3}{|c|}{ Pré-falência* } & \multicolumn{3}{|c|}{ Pós-falência ${ }^{\dagger}$} & \multicolumn{3}{|c|}{ Correção* } \\
\hline & VSP & IP & FOV & VSP & IP & FOV & VSP & IP & FOV \\
\hline $1(65)$ & 130,6 & 3,43 & $\mathrm{~T}$ & 56,7 & 1,8 & UBA & 100,93 & 4,02 & B \\
\hline $2(103)$ & 186,2 & 3,9 & $\mathrm{~T}$ & 63,16 & 2,46 & UBA & 122,34 & 3,97 & B \\
\hline $3(50)$ & 128,33 & 3,07 & $\mathrm{~T}$ & 42,11 & 1,52 & UBA & 98,44 & 3,5 & B \\
\hline $4(89)$ & 92,69 & 3,45 & B & 36,46 & 1,68 & UBA & 96,72 & 4,2 & $\mathrm{~T}$ \\
\hline $5(29)$ & 104,54 & 5,58 & $\mathrm{~T}$ & 60,02 & 0,55 & UBA & 104,7 & 3,2 & B \\
\hline $6(60)$ & 98,04 & 3,69 & $\mathrm{~T}$ & 50,32 & 3,24 & UBA & 79,27 & 3,1 & UAA \\
\hline $7(76)$ & 102,7 & 4,8 & $\mathrm{~T}$ & 48,2 & 2,12 & UBA & 75,32 & 3,3 & B \\
\hline Média & 120,94 & 3,98 & $\mathrm{~T} / \mathrm{B}$ & 50,99 & 1,91 & UBA & 96,81 & 3,61 & \\
\hline
\end{tabular}

$\mathrm{B}=$ bifásica; DAOP = doença arterial oclusiva periférica; FOV = forma da onda de velocidade de fluxo; IP = índice de pulsatilidade; $\mathrm{T}$ = trifásica; UAA = unifásica de alta aceleração; UBA = unifásica de baixa aceleração; VSP = velocidade sistólica de pico.

Medidas realizadas na artéria femoral comum.

* Pré-falência: medidas realizadas na última data de seguimento antes do diagnóstico de falência do tratamento.

†Falência: medidas realizadas na data do diagnóstico de falência do tratamento.

ॠCorreção: medidas realizadas 1 mês após a correção das estenoses ou oclusões ocorridas no grupo.

Tabela 3 - Grupo 2: medidas de análise espectral realizadas na artéria poplítea e ITB dos pacientes portadores de DAOP exclusivamente no território fêmoro-poplíteo pré e pós-tratamentos (médias)

\begin{tabular}{lccccc}
\hline Fêmoro-poplíteo & Pré $(\mathrm{n}=54)$ & Pós $(1$ mês $)(\mathrm{n}=54)$ & 6 meses $(\mathrm{n}=49)$ & 18 meses $(\mathrm{n}=46)$ & Pré/pós $(1$ mês $)(\%)$ \\
\hline ITB & 0,51 & 0,83 & 0,87 & 0,81 & 62,74 \\
VSP & 33,27 & 70,81 & 72,34 & 67,87 & 112,83 \\
IP & 2,34 & 3,80 & 3,59 & 4,45 & 62,39 \\
FOV & UBA & $\mathrm{B} / \mathrm{T}$ & $\mathrm{B} / \mathrm{T}$ & $\mathrm{B} / \mathrm{T}$ & $\mathrm{B} / \mathrm{T}$ \\
\hline
\end{tabular}

$\mathrm{B}=$ bifásica; DAOP = doença arterial oclusiva periférica; FOV = forma da onda de velocidade de fluxo; IP = índice de pulsatilidade; ITB = índice tornozelo-braço; T = trifásica; UBA = unifásica de baixa aceleração; VSP = velocidade sistólica de pico.

2) como: trifásica $(\mathrm{T})$, bifásica $(\mathrm{B})$ ou unifásica $(\mathrm{U})$. As ondas de tipo trifásicas e bifásicas eram consideradas normais. As ondas de tipo unifásica foram classificadas em dois subgrupos: as de alta aceleração (UAA, exemplo C) consideradas normais (devido à vasodilatação distal) (Figura 1, UAA) e as de baixa aceleração (UBA, Figura 1D), anormais, devido à perda de energia da onda de velocidade de fluxo, resultado de alterações hemodinamicamente significantes (estenoses ou oclusões) proximais ao local de medida. A VSP foi medida no ponto de maior velocidade da onda (Figura 1) e os índices de pulsatilidade e resistência foram calculados pelo equipamento de ultrassom. 
Tabela 4 - Grupo 2: pacientes portadores de revascularização no segmento fêmoro-poplíteo que apresentaram falência do tratamento (estenoses hemodinamicamente significantes ou oclusões) (médias)

\begin{tabular}{|c|c|c|c|c|c|c|c|c|c|}
\hline \multirow[b]{2}{*}{ Pacientes } & \multicolumn{3}{|c|}{ Pré-falência* } & \multicolumn{3}{|c|}{ Pós-falência ${ }^{\dagger}$} & \multicolumn{3}{|c|}{ Correção* } \\
\hline & VSP & IP & FOV & VSP & IP & FOV & VSP & IP & FOV \\
\hline 1 & 70,65 & 3,45 & $\mathrm{~T}$ & 17,17 & 1,92 & UBA & 68,17 & 3,87 & $\mathrm{~T}$ \\
\hline 2 & 56,67 & 4,61 & $\mathrm{~T}$ & 12,22 & 1,01 & UBA & 54,38 & 4,88 & B \\
\hline 3 & 68,86 & 2,44 & $\mathrm{~T}$ & 6,38 & 0,92 & UBA & 62,46 & 3,69 & B \\
\hline 4 & 59,89 & 5,27 & $\mathrm{~B}$ & 17,92 & 1,41 & UBA & 63,7 & 3,98 & $\mathrm{~T}$ \\
\hline 5 & 76,62 & 5,92 & $\mathrm{~T}$ & 23,61 & 1,05 & UBA & 70,2 & 4,22 & $\mathrm{~B}$ \\
\hline T6 & 77,58 & 4,35 & $\mathrm{~T}$ & 31,58 & 1,88 & UBA & 72,5 & 3,89 & $\mathrm{~T}$ \\
\hline 7 & 69,83 & 4,98 & $\mathrm{~T}$ & 17,98 & 1,02 & UBA & 66,4 & 4,7 & $\mathrm{~T}$ \\
\hline 8 & 65,29 & 5,67 & $\mathrm{~T}$ & 26,53 & 0,98 & UBA & 64,3 & 3,68 & $\mathrm{~T}$ \\
\hline Média & 68,17 & 4,58 & $1 \mathrm{~B} / 7 \mathrm{~T}$ & 19,17 & 1,27 & UBA & 61,67 & 4,14 & $3 \mathrm{~B} / 5 \mathrm{~T}$ \\
\hline
\end{tabular}

$\mathrm{AES}=$ análise espectral segmentar; $\mathrm{B}=$ bifásica FOV = forma da onda de velocidade de fluxo; $\mathrm{IP}=$ índice de pulsatilidade; $\mathrm{T}=$ trifásica; UBA = unifásica de baixa aceleração; VSP = velocidade sistólica de pico.

Medidas de AES realizadas nas artérias poplíteas.

* Pré-falência: medidas realizadas na última data de seguimento antes do diagnóstico de falência do tratamento.

†Falência: medidas realizadas na data do diagnóstico de falência do tratamento.

†Correção: medidas realizadas 1 mês após a correção das estenoses ou oclusões ocorridas no grupo.

Tabela 5 - Grupo 3: pacientes portadores de revascularização no segmento poplíteo-tibial, medidas de AES e ITB (médias)

\begin{tabular}{lccccc}
\hline Poplíteo-tibial & Pré $(\mathrm{n}=50)$ & Pós $(1$ mês $)(\mathrm{n}=50)$ & 6 meses $(\mathrm{n}=45)$ & 13 meses $(\mathrm{n}=42)$ & Pré/pós $(\%)$ \\
\hline ITB & 0,52 & 0,73 & 0,78 & 0,81 & 40,38 \\
VSP & 35,06 & 87,33 & 82,14 & 81,87 & 149,08 \\
IP & 1,70 & 2,19 & 2,98 & 2,45 & 28,8 \\
FOV & UBA & UAA/B/T & UAA/B/T & UAA/B/T & \\
\hline
\end{tabular}

$\mathrm{AES}=$ análise espectral segmentar; $\mathrm{B}=$ bifásica; FOV = forma da onda de velocidade de fluxo; $\mathrm{IP}=$ índice de pulsatilidade; ITB = índice tornozelo-braço; $\mathrm{T}$ = trifásica; UAA = unifásica de alta aceleração; UBA = unifásica de baixa aceleração; VSP = velocidade sistólica de pico.

Medidas de análise espectral realizadas nas artérias tibiais distais tratadas por enxerto ou angioplastia.

Tabela 6 - Grupo 3: pacientes portadores de revascularização no segmento poplíteo-tibial que apresentaram falência do tratamento (médias)

\begin{tabular}{|c|c|c|c|c|c|c|}
\hline & \multicolumn{3}{|c|}{ Pré-falência* } & \multicolumn{3}{|c|}{ Pós-falência ${ }^{\dagger}$} \\
\hline & VSP & IP & FOV & VSP & IP & FOV \\
\hline 1 & 70,65 & 3,45 & $\mathrm{~T}$ & 17,17 & 1,92 & UBA \\
\hline 2 & 56,67 & 4,61 & B & 12,22 & 1,01 & UBA \\
\hline 3 & 68,86 & 2,44 & $\mathrm{~T}$ & 6,38 & 0,92 & UBA \\
\hline 4 & 67,68 & 3,45 & UAA & 16,67 & 1,2 & UBA \\
\hline 5 & 71,87 & 3,67 & B & 24,35 & 1,02 & UBA \\
\hline 6 & 54,28 & 2,98 & $\mathrm{~B}$ & 21,83 & 1,01 & UBA \\
\hline Média & 64,98 & 3,34 & $2 \mathrm{~T} / 3 \mathrm{~B} / \mathrm{UAA}$ & 16,43 & 1,18 & UBA \\
\hline
\end{tabular}

$\mathrm{AES}=$ análise espectral segmentar; $\mathrm{B}=$ bifásica; FOV = forma da onda de velocidade de fluxo; $\mathrm{IP}=$ índice de pulsatilidade; $\mathrm{T}=$ trifásica; UAA = unifásica de alta aceleração; UBA = unifásica de baixa aceleração; VSP = velocidade sistólica de pico.

Medidas de AES realizadas nas artérias tibiais distais tratadas.

* Pré-falência: medidas realizadas na última data de seguimento antes do diagnóstico de falência do tratamento.

${ }^{\dagger}$ Falência: medidas realizadas na data do diagnóstico de falência do tratamento.

Correção: medidas realizadas 1 mês após a correção das estenoses ou oclusões ocorridas no grupo. 
Tabela 7 - Grupo 4: análise espectral na artéria femoral comum e ITB dos pacientes portadores de lesões hemodinamicamente significantes concomitantes no território aorto-ilíaco e fêmoro-poplíteo (médias)

\begin{tabular}{lccccc}
\hline AI-FP & Pré $(\mathrm{n}=36)$ & Pós $(1$ mês $)(\mathrm{n}=36)$ & 6 meses $(\mathrm{n}=35)$ & 12 meses $(\mathrm{n}=32)$ & Pré/pós (\%) \\
\hline ITB & 0,37 & 0,54 & 0,59 & 0,63 & 45,94 \\
VSP & 49,65 & 93,57 & 90,78 & 78,87 & 88,46 \\
IP & 1,55 & 3,51 & 3,59 & 3,67 & 126,45 \\
FOV & UBA & $\mathrm{B} / \mathrm{T}$ & $\mathrm{B} / \mathrm{T}$ & $\mathrm{B} / \mathrm{T}$ & \\
\hline
\end{tabular}

$\mathrm{AI}=$ aorto-ilíaco; $\mathrm{B}=$ bifásica; FOV = forma da onda de velocidade de fluxo; FP = fêmoro-poplíteo; IP = índice de pulsatilidade; ITB = índice tornozelo-braço; T = trifásica; UBA = unifásica de baixa aceleração; VSP = velocidade sistólica de pico.

Tabela 8 - Grupo 4: análise espectral na artéria poplítea e ITB dos pacientes portadores de lesões hemodinamicamente significantes concomitantes no território aorto-ilíaco e fêmoro-poplíteo (médias)

\begin{tabular}{lccccc}
\hline AI-FP & Pré $(\mathrm{n}=36)$ & Pós $(1$ mês $)(\mathrm{n}=36)$ & 3 meses $(\mathrm{n}=35)$ & 12 meses $(\mathrm{n}=32)$ & Pré/pós $(\%)$ \\
\hline ITB & 0,37 & 0,54 & 0,59 & 0,63 & 45,94 \\
VSP & 17,68 & 23,67 & 27,56 & 30,34 & 33,88 \\
IP & 0,91 & 1,14 & 1,34 & 1,23 & 25,27 \\
FOV & UBA & UBA & UBA & UBA & \\
\hline
\end{tabular}

$\mathrm{AI}=$ aorto-ilíaco; FOV = forma da onda de velocidade de fluxo; FP = fêmoro-poplíteo; $\mathrm{IP}=$ índice de pulsatilidade; ITB = índice tornozelo-braço; UBA = unifásica de baixa aceleração; VSP = velocidade sistólica de pico.
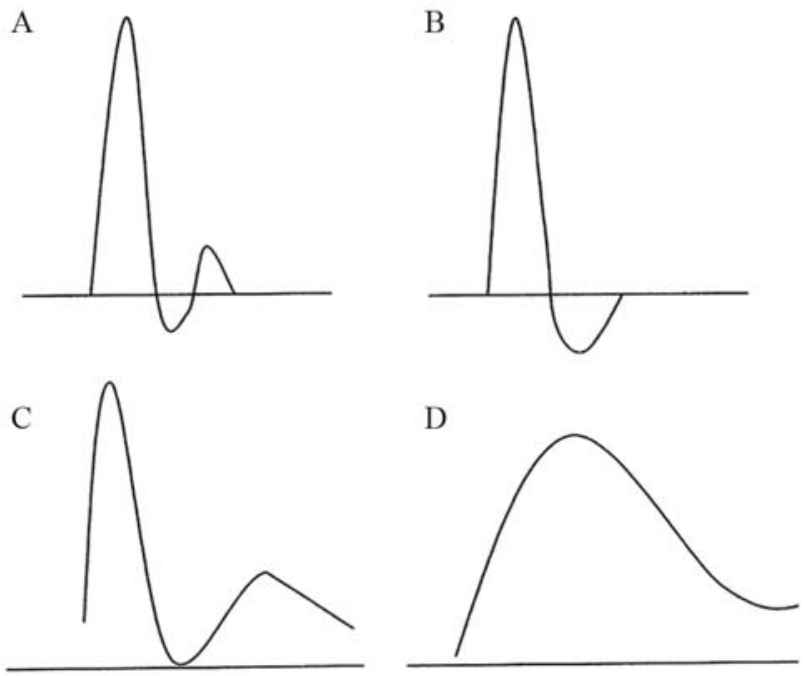

Figura 1 - Tipos de onda de velocidade de fluxo (A a D), velocidade sistólica de pico (VSP) (máxima velocidade sistólica) e índice de pulsatilidade (IP). Amplitude da onda de velocidade de fluxo dividida pela velocidade média. Tipos de onda: A) trifásica; B) bifásica; C) unifásica de alta aceleração; D) unifásica de baixa aceleração

Durante o seguimento dos pacientes, se ocorressem alterações na palpação de pulsos previamente presentes, sinais de isquemia, medidas de AES com diminuição maior que $50 \%$ nos parâmetros numéricos anteriormente medidos com mudança no tipo de onda de velocidade de fluxo (de bi ou trifásico para unifásico BA), era feito o diagnóstico provável de falência do procedimento de revascularização (enxerto ou angioplastia). O paciente era, então, submetido a exame angiográfico comprobatório. Todos os casos em que houve a suspeita de lesões hemodinamicamente significantes ou oclusão dos locais de angioplastia ou enxertos diagnosticados pela AES foram confirmados pela angiografia.

Para efeito de comparação, adicionamos um grupo de pacientes (grupo n) sem DAOP significativa, com dados demográficos semelhantes aos dos grupos estudados e composto por 102 pacientes (204 membros) que haviam sido examinados em nosso serviço com outros objetivos (como exames de carótidas, trombose venosa profunda (TVP), veias varicosas, hipertensão renovascular). Todos os pacientes desse grupo apresentavam pulsos periféricos normais, sem sopros no trajeto arterial e índice tornozelo-braço (ITB) maior que 0,9. Tal grupo foi chamado de normal (grupo n) (Tabela 9).

Foram considerados critérios de inclusão:

- Pacientes submetidos a tratamento da insuficiência arterial periférica causada por DAOP nos membros inferiores com indicação clínica de revascularização; 

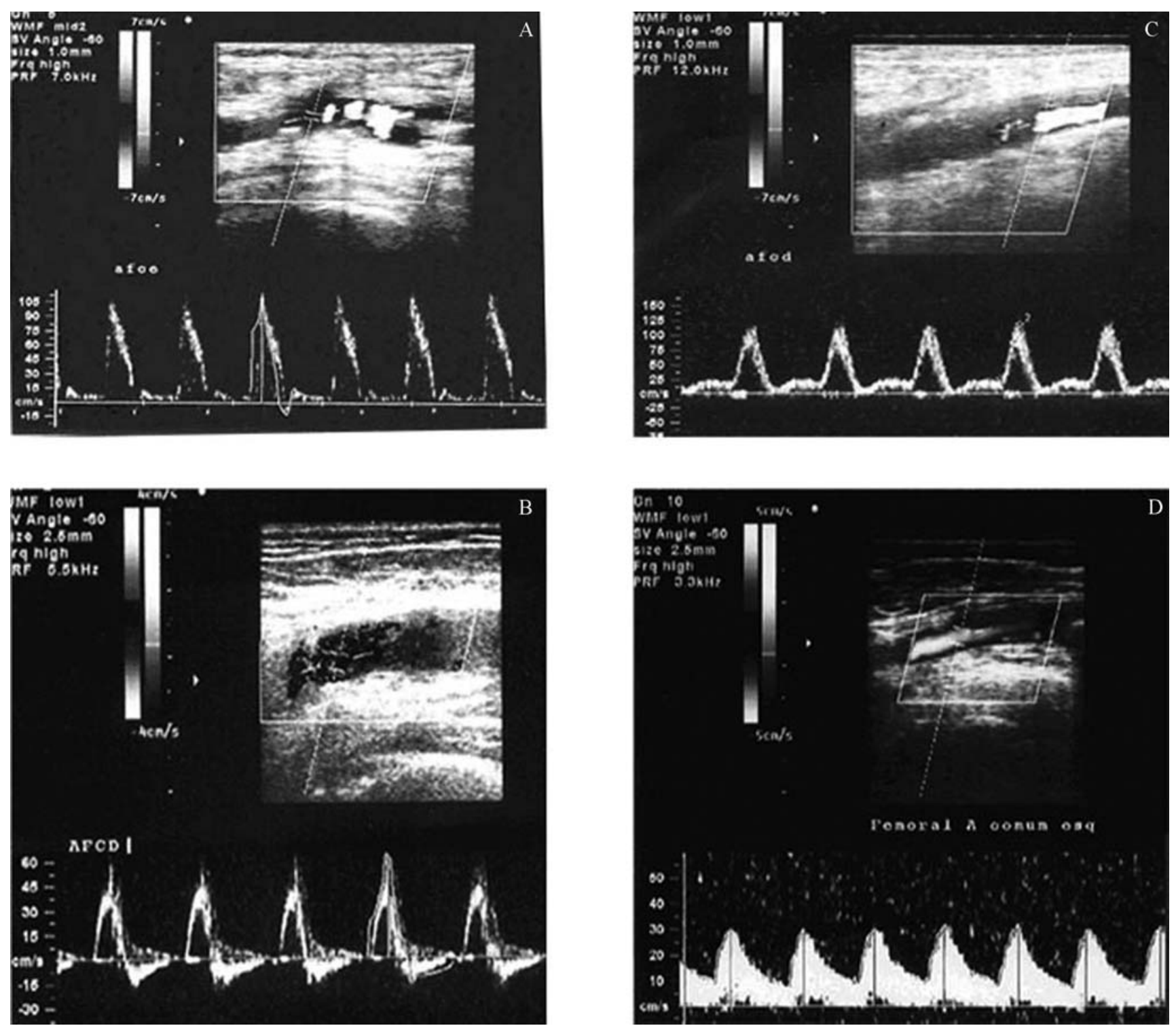

Figura 2 - Exemplos de curvas: A) curva tipo A (trifásica); B) curva tipo B (bifásica); C) curva tipo C (unifásica de alta aceleração, UAA); D) curva tipo D (unifásica de baixa aceleração, UBA)

- Pacientes com exames de ultrassom vascular pré e pós-tratamento, segundo protocolo para AES;

- Pacientes sem lesões ateroscleróticas (estenoses hemodinamicamente significantes, oclusões) na aorta torácia e abdominal;

- Pacientes portadores de lesões hemodinamicamente significativas apenas no território aorto-ilíaco, fêmoropoplíteo ou poplíteo-tibial;

- Pacientes portadores de lesões ateroscleróticas hemodinamicamente significantes concomitantemente no território aorto-ilíaco e fêmoro-poplíteo;
- Pacientes com exames angiográficos pré-tratamento completos;

- Pacientes com exames angiográficos nos casos de suspeita de lesões (estenoses hemodinamicamente significantes ou oclusões) nos enxertos e angioplastias pós-tratamento;

- Pacientes sem amputações maiores do membro examinado.

Foram considerados critérios de exclusão:

- Pacientes sem exames de ultrassom vascular pré-tratamento; 
Tabela 9 - Medidas de AES nos pacientes sem DAOP (grupo n) nos membros inferiores (médias)

\begin{tabular}{lcc}
\hline Local de medida & VSP & IP \\
\hline FC & $92,18( \pm 18,14)$ & $8,1( \pm 4,88)$ \\
Pop & $68,71( \pm 15,9)$ & $9,06( \pm 6,5)$ \\
TA & $59,81( \pm 17,78)$ & $9,56( \pm 3,71)$ \\
TP & $62,17( \pm 18,63)$ & $8,4( \pm 3,74)$ \\
\hline
\end{tabular}

$\mathrm{AES}=$ análise espectral segmentar; DAOP $=$ doença arterial oclusiva periférica; FC = artéria femoral comum; IP = índice de pulsatilidade; $\mathrm{Pop}=$ artéria poplítea; $\mathrm{TA}=$ tibial anterior; $\mathrm{TP}=$ tibial posterior; VSP = velocidade sistólica de pico.

- Pacientes com exames angiográficos não conclusivos;

- Pacientes sem exames angiográficos nos casos de suspeita de lesões (estenoses hemodinamicamente significantes ou oclusões) nos enxertos, stents e angioplastia pós-tratamento;

- Pacientes portadores de lesões hemodinamicamente significantes concomitantes no território fêmoro-poplíteo e poplíteo-tibial ou nos segmentos aorto-ilíaco, fêmoropoplíteo e poplíteo-tibial, concomitantemente;

- Pacientes com seguimento incompleto (menos um exame a cada 6 meses no período pós-tratamento);

- Pacientes portadores de aneurismas arteriais.

\section{Resultados}

No grupo de pacientes com doença exclusivamente aorto-ilíaca (grupo 1), 60 pacientes (65 procedimentos) foram seguidos por até 24 meses (média de 19,5 meses) (Tabela 1). Em tal grupo, foram realizados 11 enxertos aortoilíacos e 54 angioplastias (todas com implante de stents). A estatística aplicada ao estudo consistiu apenas da comparação entre as médias aritméticas dos parâmetros numéricos de AES, a VSP e o IP, sendo esse o objetivo do estudo.

As médias das medidas dos parâmetros numéricos de AES (VSP e IP) tomados na artéria femoral comum (artéria que define as alterações hemodinâmicas no segmento aorto-ilíaco), no grupo 1, aumentaram consistentemente em mais de $90 \%$ dos níveis pré-tratamento (pós 1 mês na Tabela 1, Figuras 3 a 5). A forma da onda de velocidade de fluxo que era unifásica (UBA na Tabela 1, pré) previamente ao tratamento tornou-se bi (B) ou trifásica (T) após a revascularização, em todos os casos (Tabela 1, em 1 mês pós-tratamento). Tais mudanças permaneceram constantes

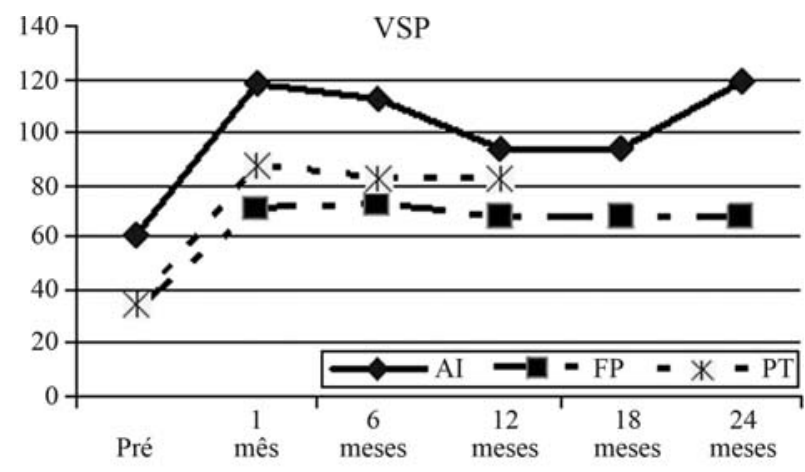

$\mathrm{AI}=$ aorto-iliaca $; \mathrm{FP}=$ fêmoro-poplitea $\mathrm{PT}=$ popliteo-tibial; VSP $=$ velocidade sistólica de pico.

Figura 3 - Medidas de VSP realizadas nos pacientes portadores de doença aorto-ilíaca, fêmoro-poplítea e poplíteo-tibial. As medidas foram feitas na artéria femoral comum dos pacientes com doença AI (grupo 1), na artéria poplítea dos pacientes com doença FP (grupo 2) e nas artérias tibiais tratadas dos pacientes com doença PT (grupo 3). Notar o padrão de aumento dos valores entre as medidas pré-tratamento (pré) e o pós-operatório imediato (1 mês), mantendo-se posteriormente no patamar $(6,12,18$ e 24 meses)

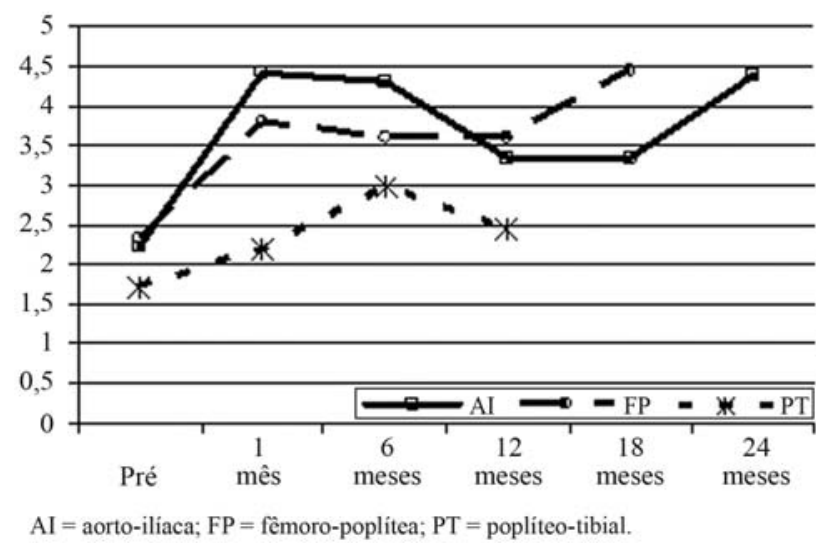

Figura 4 - Medidas de índice de pulsatilidade realizadas nos pacientes portadores de doença aorto-ilíaca, fêmoro-poplítea e poplíteo-tibial. As medidas foram feitas na artéria femoral comum dos pacientes com doença aorto-ilíaca (grupo 1), na artéria poplítea dos pacientes com doença FP (grupo 2) e nas artérias tibiais tratadas dos pacientes com doença PT (grupo 3). Notar o padrão de aumento dos valores entre as medidas pré-tratamento (pré) e o pós-operatório imediato (1 mês), mantendo-se posteriormente no patamar $(6,12,18$ e 24 meses)

durante o seguimento nos pacientes que não necessitaram de reintervenção (Tabela 1, em 6 meses, 12 meses e 24 meses).

Os aumentos no pós-operatório imediato em relação às medidas pré-tratamento foram de 92,26\% na VSP (de $61,27 \mathrm{~cm} / \mathrm{s}$ para $117,8 \mathrm{~cm} / \mathrm{s}$ ) e de $98,2 \%$ no IP (de 2,23 para 4,42) (Tabela 1, em 1 mês pós-tratamento, e Figura 5). 


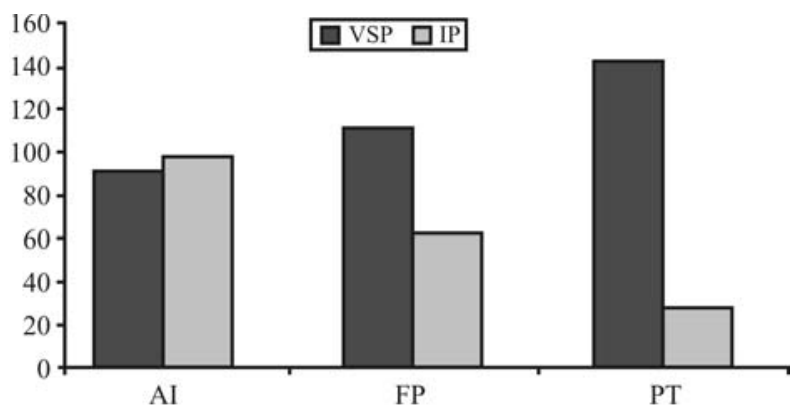

$\mathrm{AI}=$ aorto iliaca; $\mathrm{FP}=$ fêmoro-poplitea; $\mathrm{IP}=$ indice de pulsabilidade; $\mathrm{PT}=$ poplíteo-tibial; VSP $=$ velocidade sistólica de pico.

Figura 5 - Percentual de aumentos nas medidas de VSP e IP realizadas nos pacientes portadores de doença aorto-ilíaca, fêmoro-poplítea e poplíteo-tibial entre os valores pré-tratamento e o pós-operatório imediato. As medidas foram feitas na artéria femoral comum dos pacientes com doença aorto-ilíaca (grupo 1), na artéria poplítea dos pacientes com doença fêmoro-poplítea (grupo 2) e nas artérias tibiais tratadas dos pacientes com doença PT (grupo 3)

No grupo 1 ocorreram 4 oclusões e 3 estenoses (hemodinamicamente significantes) durante o seguimento, sendo que tais eventos ocorreram apenas nos pacientes tratados por angioplastia (Tabela 2). As oclusões e estenoses hemodinamicamente significativas foram consideradas como falência das revascularizações. Todos os sete pacientes apresentaram alterações significativas nos parâmetros numéricos de AES com queda dos valores da VSP para $42,16 \%$ dos valores pré-falência (de $120,94 \mathrm{~cm} / \mathrm{s}$ para $50,94 \mathrm{~cm} / \mathrm{s}$ ) e no IP para $47,98 \%$ dos níveis pré-falência (de 3,98 para 1,91) (Tabela 2, Figuras 6 a 8). As formas da onda de velocidade de fluxo que eram bi ou trifásicas após o tratamento tornaram-se unifásicas (UBA) em todos os casos de falência no tratamento (Tabela 2, pós-falência, UBA). Todos os sete pacientes foram tratados das oclusões e estenoses por novas angioplastias e apresentaram aumento significativo nos parâmetros numéricos e retorno da forma da onde de velocidade de fluxo para padrões bi ou trifásicos (Tabela 2, Figuras 6 a 8). A VSP aumentou $89,86 \%$ após as angioplastias pós-falência (de $50,99 \mathrm{~cm} / \mathrm{s}$ para $96,81 \mathrm{~cm} / \mathrm{s})$. O IP aumentou $89 \%$ depois da angioplastia pós-falência (de 1,91 para 3,61) (Tabela 2, Figura 8).

O grupo de pacientes com doença exclusivamente no território fêmoro-poplíteo (grupo 2) composto por 54 pacientes (54 procedimentos) foi seguido por até 18 meses (13,2 meses em média) (Tabela 3, Figuras 3 a 5). Os pacientes foram tratados por enxertos ( 23 casos) ou por angioplastia com stents (31 casos). Nestes casos, houve aumento

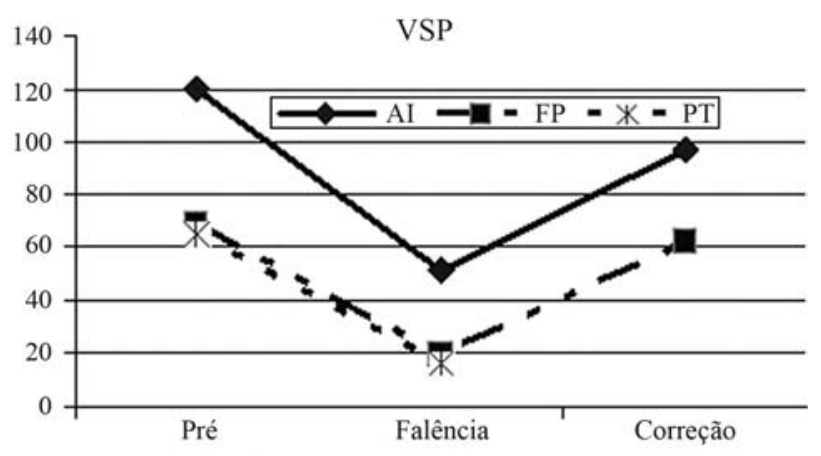

$\mathrm{AI}=$ aorto-iliaca; $\mathrm{FP}=$ fêmoro poplitea $; \mathrm{PT}=$ popliteo-tibial;

$\mathrm{VSP}=$ velocidade sistólica de pico.

Figura 6 - Medidas de VSP realizadas nos pacientes portadores de doença aorto-ilíaca, fêmoro-poplítea e poplíteo-tibial antes da falência (Pré), após a falência (Falência) e após a correção (Correção). As medidas foram feitas na artéria femoral comum dos pacientes com doença aorto-ilíaco (grupo 1), na artéria poplítea dos pacientes com doença fêmoro-poplítea (grupo 2) e nas artérias tibiais tratadas dos pacientes com doença PT (grupo 3)

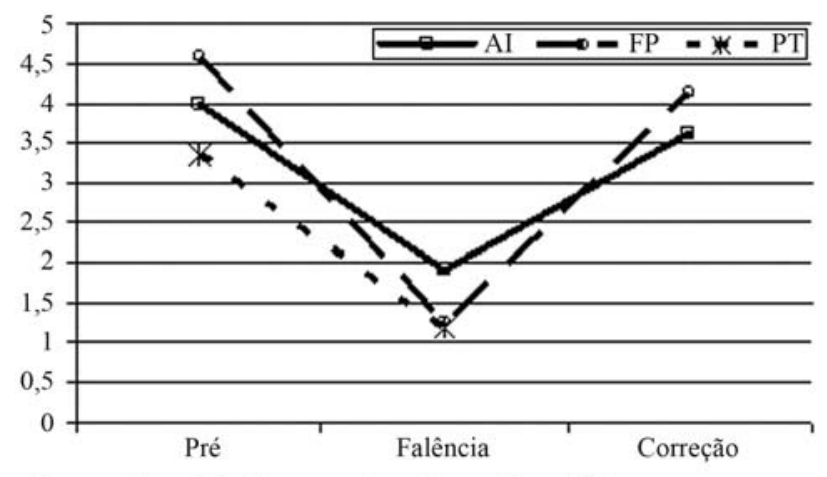

$\mathrm{AI}=$ aorto-iliaca; $\mathrm{FP}=$ fêmoro poplitea $\mathrm{PT}=$ popliteo-tibial.

Figura 7 - Medidas de índice de pulsatilidade realizadas nos pacientes portadores de doença aorto-ilíaca, fêmoro-poplítea e poplíteo-tibial antes da falência (Pré), após a falência (Falência) e após a correção (Correção). As medidas foram feitas na artéria femoral comum dos pacientes com doença aorto-ilíaca (grupo 1), na artéria poplítea dos pacientes com doença fêmoro-poplítea (grupo 2) e nas artérias tibiais tratadas dos pacientes com doença poplíteo-tibial (grupo 3)

significativo e consistente nos parâmetros numéricos da AES medidos na artéria poplítea (que define as condições hemodinâmicas do segmento fêmoro-poplíteo), comparados aos valores pré-tratamento (Tabela 3, Figuras 3 a 5). Os aumentos foram de $112,83 \%$ na VSP (de $33,27 \mathrm{~cm} / \mathrm{s}$ para $70,81 \mathrm{~cm} / \mathrm{s}$ ) e de $62,39 \%$ no IP (de 2,34 para 3,8) (Figuras 5 e 9) com mudança da forma da onda de velocidade de fluxo de unifásico (UBA) para bi ou trifásico em todos os casos (Tabela 3).

No grupo 2, ocorreram 6 oclusões nos pacientes tratados por angioplastia e 2 estenoses hemodinamicamente 


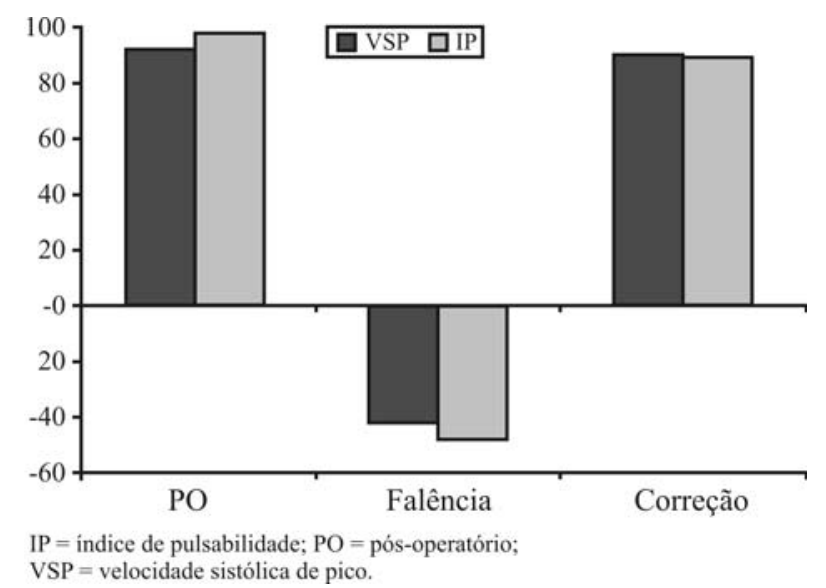

Figura 8 - Mudanças percentuais nos valores de VSP e IP no grupo dos pacientes com doença apenas no território aorto-ilíaco durante o pós-operatório (PO), quando ocorreu falência do tratamento (Falência) e após a correção (Correção)

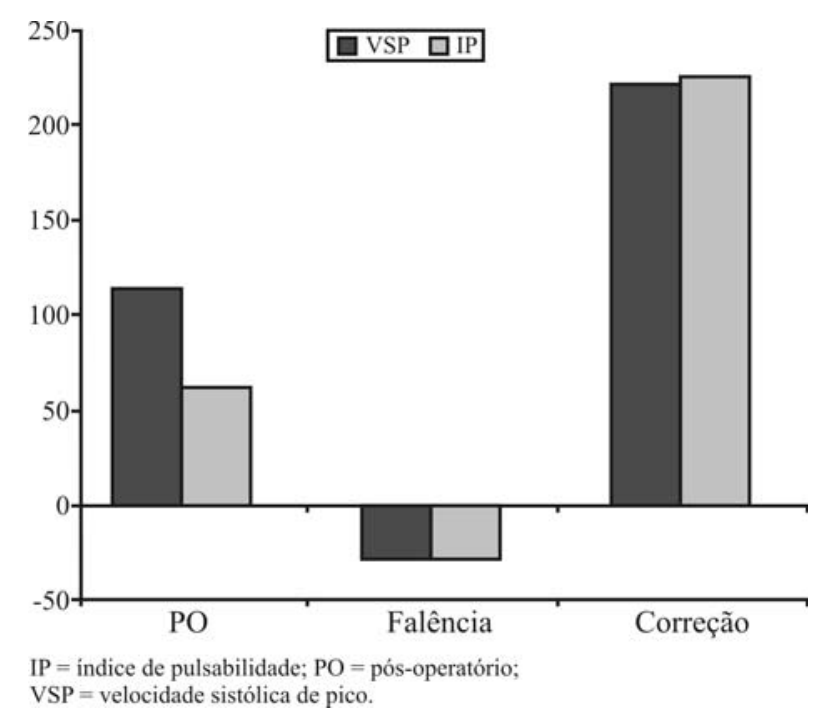

Figura 9 - Mudanças percentuais nos valores de VSP e IP no grupo dos pacientes com doença apenas no território fêmoro-poplíteo durante o pós-operatório (PO), quando ocorreu falência do tratamento (Falência) e após a correção (Correção)

significantes dentre os tratados por enxertos. Aqui também as oclusões e estenoses hemodinamicamente significantes foram consideradas como falência da revascularização. Em tais pacientes, a VSP e o IP medidos na artéria poplítea apresentaram grandes alterações. A VSP diminuiu em média para $28,12 \%$ dos valores pré-falência (de $68,17 \mathrm{~cm} / \mathrm{s}$ para $19,17 \mathrm{~cm} / \mathrm{s}$ ) e o IP diminuiu em média para $27,73 \%$ dos valores pré-falência (de 4,58 para 1,27) (Tabela 4, Figuras 6,7 e 9). A forma da onda de velocidade de fluxo também mudou de bi ou trifásica para unifásica (UBA) em todos os pacientes (Tabela 4). Quando tratados por nova revascularização (enxertos e correção das estenoses), as medidas realizadas na artéria poplítea mostraram comportamento semelhante ao ocorrido com a revascularização inicial com aumento da VSP e IR e mudança na forma da onda de velocidade de fluxo de unifásica (UBA) para bi ou trifásica (Tabela 4, Figuras 6 a 9). Nesses casos, a VSP aumentou $221,7 \%$ (de $19,17 \mathrm{~cm} / \mathrm{s}$ para $61,67 \mathrm{~cm} / \mathrm{s})$ e o IP aumentou 225\% (de 1,27 para 4,14) (Figuras 6, 7 e 9).

Os pacientes com doença apenas no território poplíteo-tibial (grupo 3), composto de 44 pacientes (50 procedimentos) foram acompanhados por um tempo médio de 13,47 meses (até 15 meses) (Tabela 5, Figuras 3 a 5). Nesse grupo, 41 membros foram tratados por enxertos e 9 membros foram tratados por angioplastia.. As medidas de AES usadas para comparações pré e pós-tratamentos referem-se apenas às tomadas nas artérias tibiais tratadas (anterior ou posterior).

No grupo 3, os parâmetros numéricos de AES (VSP e IP) e a forma da onda de velocidade de fluxo após os tratamentos comportaram-se da mesma maneira que nos dois grupos anteriores (Tabela 5, Figuras 3 a 5). Houve aumento de $149,08 \%$ na VSP (de $35,06 \mathrm{~cm} / \mathrm{s}$ para $87,33 \mathrm{~cm} / \mathrm{s}$ ) e de $28,8 \%$ na IP (de 1,7 para 2,19) (Tabela 5, Figuras 3 a 5) e a forma da onda de velocidade de fluxo mudou de unifásica (UBA) para bi ou trifásica na maioria dos casos (em um caso para UAA) (Tabela 5). Na eventualidade de oclusão dos enxertos os parâmetros de AES comportaram-se da mesma maneira que nos casos anteriores (pacientes com doença no exclusivamente no segmento aorto-ilíaco ou fêmoro-poplíteo) com queda na VSP, no IP e alteração da forma da onda de velocidade de fluxo de bi ou trifásicas para unifásica (UAB) (Tabela 6, Figuras 6 e 7). A VSP apresentou uma diminuição de $74,16 \%(64,98 \mathrm{~cm} / \mathrm{s}$ para $16,43 \mathrm{~cm} / \mathrm{s}$ ) e o IP de $64,67 \%$ (de 3,34 para 1,18 ).

No grupo de pacientes com doença concomitante no território aorto-ilíaco e fêmoro-poplíteo (grupo 4), 35 pacientes (36 procedimentos) foram acompanhados por tempo médio de 15,2 meses (até 16 meses). Neste grupo foram incluídos apenas pacientes que foram submetidos à correção da doença no território aorto-ilíaco (Tabelas 7 e 8). Os pacientes com revascularização sequencial (aorto-ilíaca e fêmoro-poplítea) foram excluídos, pois eram apenas 3 pacientes. As medidas feitas na artéria femoral comum dos pacientes com revascularização proximal (aorto-ilíaca) mostraram aumento nos parâmetros numéricos da AES de 
$88,46 \%$ na VSP e $126,45 \%$ na IP com mudanças nos padrões de forma da onda de velocidade de fluxo de unifásicos (UBA) para bi ou trifásicos (Tabela 7). Contudo, nas medidas realizadas nas artérias poplíteas (onde não houve revascularização do segmento), os aumentos de VSP e IP foram de apenas 33,8 e $25,27 \%$ respectivamente, sem alterações da forma da onda de velocidade de fluxo (Tabela 8).

\section{Discussão}

O uso de ultrassom vascular para diagnóstico e planejamento do tratamento da DAOP teve sua capacidade diagnóstica e acurácia demonstrada em vários trabalhos ${ }^{1-12}$, tanto nos segmentos supra quanto os infrainguinais sendo seu uso hoje considerado parte da propedêutica vascular.

O seguimento dos pacientes tratados por revascularização do membro (através de enxertos ou tratamentos endovasculares) usando a ultrassom vascular tem-se mostrado um método confiável e seguro ${ }^{14-23}$. O seguimento de enxertos usando o exame ultrassom vascular pode inclusive aumentar a perviedade primária chamada de perviedade assistida $^{19-23}$. Em pacientes portadores de enxertos usando veias braquiais, o exame de seguimento por ultrassom vascular demonstrou que mais de $40 \%$ dos enxertos necessitavam de intervenções no pós-operatório, para manutenção da sua perviedade ${ }^{24}$. Em outra série $e^{25}$, o seguimento de enxertos fêmoro-femoral usando ultrassom vascular aumentou a perviedade (primária) de 86\% (em 1 ano), 78\% (em 3 anos) e $62 \%$ (em 5 anos) para uma perviedade assistida de $95 \%$ ( 1 ano) e $88 \%$ ( 3 e 5 anos). Também foi demonstra$\mathrm{do}^{26}$ que o seguimento pós-operatório de enxertos infrainguinais para tratamento de isquemia crítica usando o ultrassom vascular tem um custo menor e resultam em menos amputações que o seguimento baseado em ITB ou baseado apenas em exames clínicos.

Contudo, nos protocolos clássicos para seguimento dos tratamentos usando ultrassom vascular, examina-se toda a extensão do membro incluindo a artéria doadora e a receptora (nos enxertos) ou o local de angioplastia (nos tratamentos endovasculares). O exame é realizado em modo color com medidas de velocidades em pontos indicados pelas alterações da cor ${ }^{14,19,24,27,28}$ em repouso e eventualmente após exercício físico ou hiperemia reativa ${ }^{3}$. As estenoses são graduadas pela correlação (ratio) da VSP proximal e no local de lesão. As medidas de VSP para calculo desta correlação devem ter o ângulo de insonação corrigi- do e algumas vezes isto é difícil de ser realizado, como no segmento aorto-ilíaco, onde as artérias do segmento apresentam uma tortuosidade natural. A correção de angulo também pode ser dificultada nos locais de anastomoses. Outras causas de dificuldade de medida das correlações da VSP nas artérias incluem a massa muscular no segmento fêmoro-poplíteo, gases intra-abdominais, lesões na origem de artérias, bem como a eventual desproporção nos diâmetros entre os enxertos e artérias doadoras ou receptoras, além de segmentos com placas ateroscleróticas calcificadas, que não permitem a insonação local. Todas essas situações podem ser complicadas pelo nível de experiência do examinador ${ }^{29}$. Por outro lado, a medida do aumento relativo (ratio) da VSP em uma estenose não permite a avaliação de estenoses sequenciais ${ }^{11}$.

Existem discrepâncias entre as medidas para definição do gradiente de pressão presente em stents pós-tratamento quando se comparam medidas de pressão tomadas com cateteres e alterações de VSP intraluminares ${ }^{9}$, sendo que isso se deve, provavelmente, ao posicionamento dos cateteres ou angulação do eixo de fluxo em relação ao feixe de ultrassom e turbulência intraluminares. Também foi demonstrado que as medidas de VSP em enxertos geralmente não apresentam um padrão fixo, dificultando também sua aplicação comparativa temporal ${ }^{29}$. Todas essas dificuldades podem ser minimizadas no exame utilizando a técnica de AES, porque a medidas são realizadas em locais relativamente distantes do ponto de inserção do stent ou mesmo das anastomoses dos enxertos ${ }^{13}$.

Várias maneiras para análise da hemodinâmica arterial foram usadas por outros como a medida local de $\mathrm{VSP}^{24,25,27,30-32}$, do IP $\mathrm{IP}^{33,34}$, do índice de resistência (IR) ${ }^{33,34}$ ou a análise da forma da onda de velocidade de fluxo ${ }^{6,30,31,35}$. A diminuição na VSP comparada a uma medida prévia em um mesmo segmento arterial foi usada para indicar a presença de estenose hemodinamicamente significante proximal ao local da medida em enxertos usando veias braquiais ${ }^{24}$. Por outro lado, VSP baixas no terço médio de enxertos de politetrafluoretileno (PTFE) $)^{30,31}$ já foram correlacionadas à falência de tais enxertos, mas o parâmetro não foi analisado nem temporalmente nem de maneira segmentar.

Em trabalho usando pacientes tratados de hipertensão renovascular ${ }^{32}$ por revascularização renal (enxerto ou angioplastia), a VSP medida no parênquima renal (portanto 
distal ao local de tratamento) aumentou $42 \%$ no póstratamento em relação às medidas pré-tratamento. Outro trabalho recente ${ }^{25}$ sobre o seguimento de enxertos fêmoro-femorais mostra que em enxertos nos quais estenoses proximais hemodinamicamente significantes desenvolveram-se pós-operatoriamente, a VSP era menor que $60 \mathrm{~cm} / \mathrm{s}$ em cinco de oito casos, sendo que tal VSP triplicou após correção da estenose proximal. Relato de baixas velocidades de fluxo (VSP) medidas em um ponto qualquer de um enxerto pode ser um indicativo de tendência à oclusão ${ }^{27}$.

Também já foi demonstrado que os índices de pulsatilidade e de resistência, além dos índices de aceleração, medidos nas artérias intrarrenais em pacientes portadores de estenoses das artérias renais aumentam e retornam aos níveis de normalidade após tratamento por angioplastia ${ }^{33}$. Nos pacientes em que estenose dos stents ocorreu após o tratamento (reestenose) os parâmetros voltaram a diminuir para níveis pré-tratamento ${ }^{33}$. Após tratamentos endovasculares em pacientes portadores de transplantes renais com estenoses nas artérias aferentes, o IR aumentou distalmente de modo expressivo ${ }^{34}$. A melhora da hemodinâmica local foi confirmada por testes funcionais e angiografia.

$\mathrm{Na}$ análise da forma da onda de velocidade de fluxo, ondas monofásicas são consideradas como padrão de fluxo anormal, principalmente as de baixa aceleração, e foram definidas em vários estudos como indicativo de doença oclusiva significante proximalmente ao local de exa$\mathrm{me}^{6,30,31}$. Outros usaram fórmula que combina VSP, IR e a forma da onda de velocidade de fluxo medida na artéria femoral comum para diagnosticar doença significativa no território aorto-ilíaco ${ }^{12}$. Medidas de VSP e da forma da onda de velocidade de fluxo geradas por ressonância magnéticas comportaram-se de maneira semelhante às produzidas pelo ultrassom ${ }^{35}$.

Em nosso trabalho, medimos parâmetros de AES (VSP, IP e forma da onda de velocidade de fluxo) de maneira segmentar, nas artérias femorais comuns, poplíteas e tibiais de pacientes portadores de DAOP pré e pós-tratamentos (grupos 1 a 4) (Tabelas 1 a 8 ) que mostraram grande correlação com os achados angiográficos em trabalho anterior $^{13}$. Tais dados foram também comparados às medidas de AES realizadas em pacientes sem doença significativa (grupo n) (Tabela 9), confirmando que os valores desses parâmetros eram consistentemente diferentes dos valores medidos nas artérias correspondentes nos pacientes sem DAOP.

Após o revascularização dos membros com isquemia arterial causada pela DAOP, os valores numéricos dos parâmetros de AES (VSP e IP) medidos no segmento distalmente à artéria receptora (ou distalmente ao segmento tratado por angioplastia), aumentaram significativamente, e assim permaneceram enquanto a revascularização permanecesse pérvia (Tabelas 1, 3 e 5, Figuras 3 e 4). A VSP aumentou mais de 90\% (Tabelas 1, 3 e 5, Figura 5) após a revascularização, indicando aumento significativo na energia cinética da onda de velocidade de fluxo ${ }^{2,36}$. Quanto ao IP, seu aumento foi de $98 \%$ após tratamento das lesões no segmento aorto-ilíaco, de $62 \%$ após tratamentos no segmento fêmoro-poplíteo e de $28,8 \%$ após tratamentos no segmento poplíteo-tibial. Essas diferenças podem ter ocorrido devido à vasodilatação distal, colaterais ou alterações na complacência da parede arterial. A forma da onda de velocidade de fluxo também mudou o padrão (Figuras $1 \mathrm{e}$ 2) de unifásica de baixa aceleração (UBA) no pré-tratamento para bi ou trifásica.

Quando ocorria falência do tratamento (oclusão ou estenose hemodinamicamente significante dos enxertos ou angioplastias) os índices numéricos de AES medidos no segmento distalmente à artéria receptora (ou distalmente ao segmento tratado por angioplastia), diminuíram significativamente, e a forma da onda de velocidade de fluxo mudou voltando aos padrões unifásicos (UBA) (Tabelas 2, $4 \mathrm{e}$ 6, Figuras 6 a 9). A VSP e o IP diminuíram em todos os grupos. Caíram mais de $40 \%$ dos valores pré-falência nos pacientes tratados por lesões do segmento aorto-ilíaco, 28 e $27 \%$, respectivamente, nos pacientes com lesões fêmoro-poplíteas e 25 e $35 \%$, respectivamente, nos portadores de lesões poplíteo-tibiais. Nos pacientes tratados com correção das estenoses ou novas revascularizações (correção, nas Tabelas 3 e 5, Figuras 6 a 9), houve aumento de VSP e IP tanto no segmento aorto-ilíaco (aumento de 89\%) (Tabela 2) como no fêmoro-poplíteo (aumento maior que $220 \%$ ) (Tabela 4). A forma da onda de velocidade de fluxo mudou de unifásica para bi ou trifásica em todos os casos em que houve correção da falência de tratamento (Tabelas 2 e 5).

Em termos de tempo para realização de um exame no modo clássico de exame ultrassom vascular, relatos mostram que o exame demora em torno de 1 hora $^{8}$, mesmo em 
serviços com examinadores bem treinados. O tempo gasto nos exames deve ser maior nos serviços onde residentes em treinamento realizam os exames. No caso do exame de AES, o tempo de exame é muito diminuído porque são necessárias apenas três ou quatro medidas em um membro. As vantagens da AES são várias, além do menor tempo para sua realização. Com a AES, é possível examinar pacientes com tratamentos em locais menos acessíveis ao ultrassom, como abdome, canal dos adutores, terço proximal da perna. Também é possível definir valores numéricos de fácil entendimento e que podem ser usados na comparação temporal, muito útil no seguimento. Nos casos em que as medidas de AES em repouso deixem dúvidas, sempre é possível realizá-la após hiperemia reativa ou exercício.

Para comparação de nossos resultados, preferimos usar as percentagens de alterações dos valores numéricos de AES porque sentimos que tal valor define diretamente a alteração na hemodinâmica local, por possibilitarem o uso na comparação temporal, por serem mais facilmente assimiláveis e não dependerem de valores absolutos para definição (Figuras 8 e 9). Quanto à medida da VSP de maneira absoluta, sabemos que essa apresenta alterações quando medida em local onde a luz arterial esteja alterada, podendo sofrer influência da hemodinâmica cardíaca, e que também tem uma relação inversamente proporcional com o diâmetro do vaso, pois quanto maior o diâmetro de um vaso menor será a velocidade do sangue quando o fluxo é constante.

O aumento das medidas numéricas (VSP, IP) da AES e a transformação da forma da onda de velocidade de fluxo de uni para bi ou mesmo trifásicas, juntamente com os sinais clínicos de melhora da perfusão local (cicatrização de lesões tróficas, abolição da dor de repouso) e aumento do ITB é uma demonstração de que a hemodinâmica local mudou para padrões semelhantes aos de pacientes sem DAOP significante (Tabela 9). Portanto, sem lesões hemodinamicamente significativas. A manutenção de tais parâmetros de AES enquanto os tratamentos (enxertos, angioplastia e stents) estavam pérvios e a queda desses quando os tratamentos entravam em falência com o posterior aumento de novo nos parâmetros quando o problema era sanado confirma que esses podem ser usados como padrões de revascularização, principalmente de maneira temporal.

\section{Conclusões}

As alterações consistentes dos valores numéricos da AES e da forma da onda de velocidade de fluxo quando os tratamentos eram bem-sucedidos e quando não havia falência, retornando aos padrões pós-tratamento quando nova revascularização era realizada, mostram que a AES pode ser usada como instrumento de acompanhamento da DAOP dos membros inferiores. Isso permite um diagnóstico precoce e não-invasivo de eventuais estenoses hemodinamicamente significantes e oclusões de procedimentos cirúrgicos realizados. A correlação entre tais medidas e alterações da hemodinâmica local e os diferentes arranjos das lesões ateroscleróticas podem ser inferidos pelo comportamento das medidas de AES pré e pós-tratamentos.

\section{Referências}

1. Katsamouris AN, Giannoukas AD, Tsetis D, Kostas T, Petinarakis I, Gourtsoyiannis N. Can ultrasound replace arteriography in the management of chronic arterial occlusive disease of the lower limb? Eur J Vasc Endovasc Surg. 2001;21:155-9

2. Shaalan WE, French-Sherry E, Castilla M, Lozanski L, Bassiouny HS. Reliability of common femoral artery hemodynamics in assessing the severity of aortoiliac inflow disease. J Vasc Surg. 2003;37:960-9.

3. Coffi SB, Ubbink DT, Zwiers I, van Gurp JA, Legemate DA. Improved assessment of the hemodynamic significance of borderline iliac stenosis with use of hyperemic duplex scanning. J Vasc Surg. 2002;36:575-80.

4. Ascher E, Salles-Cunha SX, Marks N, Hingorani A. Lower extremity arterial mapping: duplex ultrasound as an alternative to arteriography prior to femoral and popliteal reconstruction. In: AbuRahma AF, Bergan JJ, editors. Noninvasive vascular diagnosis: a practical guide to therapy. $2^{\mathrm{a}}$ ed. London: Springer; 2007. p. 293-302.

5. Lowery AJ, Hynes N, Manning BJ, Mahendran M, Tawfik S, Sultan S. A prospective feasibility study of duplex ultrasound arterial mapping, digital-subtraction angiography, and magnetic resonance angiography in management of critical lower limb ischemia by endovascular revascularization. Ann Vasc Surg. 2007;21:443-51.

6. Spronk S, den Hoed PT, de Jonge LC, van Dijk LC, Pattynama PM. Value of the duplex waveform at the common femoral artery for diagnosing obstructive aortoiliac disease. J Vasc Surg. 2005;42:236-42; discussion 242.

7. Norgren L, Hiatt WR, Dormandy JA, Nehler MR, Harris KA, Fowkes FG; TASC II Working Group. Inter-society consensus for the management of peripheral arterial disease (TASC II). J Vasc Surg. 2007;45 Suppl S:S5-67.

8. Proia RR, Walsh DB, Nelson PR, et al. Early results of infragenicular revascularization based solely on duplex arteriography. J Vasc Surg. 2001;33:1165-70.

9. De Smet AA, Tetteroo E, Moll FL. Noninvasive evaluation before and after percutaneous therapy of iliac artery stenoses: 
the value of the Bernoulli-predicted pressure gradient. J Vasc Surg. 2000;32:153-9.

10. AbuRahma AF, Jarrett KS. Segmental doppler pressures and doppler waveform analysis in peripheral vascular disease of the lower extremities. In: AbuRahma AF, Bergan JJ, editors. Noninvasive vascular diagnosis: a practical guide to therapy. $2^{\mathrm{a}}$ ed. London: Springer; 2007. p. 231-44.

11. Bertolotti C, Qin Z, Lamontagne B, Durand LG, Soulez G, Cloutier G. Influence of multiple stenosis on echo-Doppler functional diagnosis of peripheral arterial disease: a numerical and experimental study. Ann Biomed Eng. 2006;34:56474.

12. Fontcuberta J, Flores A, Langsfeld M, et al. Screening algorithm for aortoiliac occlusive disease using duplex ultrasonography-acquired velocity spectra from the distal external iliac artery. Vascular. 2005;13:164-72.

13. de Morais Filho D, Miranda F Jr, Del Carmen Janeiro Peres M, Barros N Jr, Buriham E, Salles-Cunha SX. Segmental waveform analysis in the diagnosis of peripheral arterial occlusive diseases. Ann Vasc Surg. 2004;18:714-24.

14. Bandyk DF. Duplex ultrasound surveillance can be worthwhile after arterial intervention. Perspect Vasc Surg Endovasc Ther. 2007;19:354-9; discussion 360-1.

15. Avino AJ, Bandyk DF, Gonsalves AJ, et al. Surgical and endovascular intervention for infrainguinal vein graft stenosis. J Vasc Surg. 1999;29:60-70; discussion 70-1.

16. Armstrong PA, Bandyk DF, Wilson JS, Shames ML, Johnson BL, Back MR. Optimizing infrainguinal arm vein bypass patency with duplex ultrasound surveillance and endovascular therapy. J Vasc Surg. 2004;40:724-30; discussion 730-1.

17. Stone PA, Armstrong PA, Bandyk DF, et al. The value of duplex surveillance after open and endovascular popliteal aneurysm repair. J Vasc Surg. 2005;41:936-41.

18. Back MR, Novotney M, Roth SM, et al. Utility of duplex surveillance following iliac artery angioplasty and primary stenting. J Endovasc Ther. 2001;8:629-37.

19. Mills JL Sr, Wixon CL, James DC, Devine J, Westerband A, Hughes JD. The natural history of intermediate and critical vein graft stenosis: recommendations for continued surveillance or repair. J Vasc Surg. 2001;33:273-80; discussion 278-80.

20. Ferris BL, Mills JL Sr, Hughes JD, Durrani T, Knox R. Is early postoperative duplex scan surveillance of leg bypass grafts clinically important? J Vasc Surg. 2003;37:495-500.

21. Giswold ME, Landry GJ, Sexton GJ, et al. Modifiable patient factors are associated with reverse vein graft occlusion in the era of duplex scan surveillance. J Vasc Surg. 2003;37:47-53.

22. Carter A, Murphy MO, Halka AT, et al. The natural history of stenoses within lower limb arterial bypass grafts using a graft surveillance program. Ann Vasc Surg. 2007;21:695703.

23. Bandyk DF. Surveillance after lower extremity arterial bypass. Perspec Vasc Surg Endovasc Ther. 2007;19:376-83; discussion 384-5.
24. Armstrong PA, Bandyk DF, Wilson JS, Shames ML, Johnson BL, Back MR. Optimizing infrainguinal arm vein bypass patency with duplex ultrasound surveillance and endovascular therapy. J Vasc Surg. 2004;40:724-30; discussion 730-1.

25. Stone PA, Armstrong PA, Bandyk DF, et al. Duplex ultrasound criteria for femorofemoral bypass revision. J Vasc Surg. 2006;44:496-502.

26. Visser K, Idu MM, Buth J, Engel GL, Hunink MG. Duplex scan surveillance during the first year after infrainguinal autologous vein bypass grafting surgery: costs and clinical outcomes compared with other surveillance programs. J Vasc Surg. 2001;33:123-30.

27. Bandyk DF. Infrainguinal vein bypass graft surveillance: how to do it, when to intervene and it is cost effective? J Am Coll Surg. 2002;194(1 Suppl):S40-52.

28. Taggert JB, Kupinski AM, Darling RC 3rd, Trub M, Paty PS. Hemodynamic changes associated with bypass stenosis regression. J Vasc Surg. 2005;41:1013-7.

29. Lui EY, Steinman AH, Cobbold RS, Johnston KW. Human factors as a source of error in peak Doppler velocity measurement. J Vasc Surg. 2005;42:972-9.

30. Brumberg RS, Back MR, Armstrong PA, et al. The relative importance of graft surveillance and warfarin therapy in infrainguinal prosthetic bypass failure. J Vasc Surg. 2007;46:1160-6.

31. Calligaro KD, Doerr K, McAffee-Bennett S, Krug R, Raviola CA, Dougherty MJ. Should duplex ultrasonography be performed for surveillance of femoropopliteal and femorotibial arterial prosthetic bypasses? Ann Vasc Surg. 2001;15:520-4.

32. Cohn EJ Jr, Benjamin ME, Sandager GP, Lilly MP, Killewich LA, Flinn WR. Can intrarenal duplex waveform analysis predict successful renal artery revascularization? J Vasc Surg. 1998;28:471-80; discussion 480-1.

33. Marana I, Airoldi F, Burdick L, et al. Effects of balloon angioplasty and stent implantation on intrarenal echo-Doppler velocimetric indices. Kidney Int. 1998;53:1795-800.

34. Ruggenenti P, Mosconi L, Bruno S, et al. Post-transplant renal artery stenosis: the hemodynamic response to revascularization. Kidney Int. 2001;60:309-18.

35. Wikström J, Johansson L, Karacagil S, Ahlström H. Correlation of femoral artery flow velocity waveform with ipsilateral iliac artery stenoses assessed with magnetic resonance imaging. Acta Radiol. 2007;48:422-30.

36. Strandness DE, Sumner DS. The effect of geometry on arterial blood flow. In: Strandness DE, Sumner DS. Hemodynamics for surgeons. New York: Grune \& Stratton; 1975. p. 96-119.

Correspondência:

Domingos de Morais Filho

Avenida Bandeirantes, 403

CEP 86010-020 - Londrina, PR

E-mail: domingos.morais@sercomtel.com.br 